\title{
Overexpression and Enzymatic Assessment of Antigenic Fragments of Hyaluronidase Recombinant Protein From Streptococcus pyogenes
}

\author{
Shabnam Sadoogh Abbasian ${ }^{1}$; Ehsanollah Ghaznavi $\operatorname{Rad}^{2}$; Neda Akbari ${ }^{3}$; Mohammad Reza \\ Zolfaghari ${ }^{4}$; Iraj pakzad ${ }^{5}$; Hamid Abtahi ${ }^{1, "}$ \\ ${ }_{1}^{1}$ Molecular and Medicine Research Center, Arak University of Medical Sciences, Arak, IR Iran \\ ${ }^{2}$ Department of Microbiology and Immunology, School of Medicine, Arak University of Medical Sciences, Arak, IR Iran \\ ${ }^{3}$ Department of Microbiology, Faculty of Science, Arak branch, Islamic Azad University, Arak, IR Iran \\ ${ }_{5}^{4}$ Department of Microbiology, Qom Branch, Islamic Azad University, Qom, IR Iran \\ ${ }_{5}^{5}$ Clinical Microbiology Research Center, Ilam University of Medical Sciences, Ilam, IR Iran \\ ${ }^{*}$ Corresponding author: Hamid Abtahi, Molecular and Medicine Research Center, Arak University of Medical Sciences, Arak, IR Iran. Tel: +98-8612227109, Fax: +98-8612227136, E-mail: \\ abtahi@arakmu.ac.ir
}

Received: July 17, 2013; Revised: June 3, 2014; Accepted: June 5, 2014

\begin{abstract}
Background: Hyaluronidase catalyzes the hydrolysis of hyaluronan polymers to N-acetyl-D-glucosamine and D-glucuronic acid. This enzyme is a dimer of identical subunits. Hyaluronidase has different pharmaceutical and medical applications. Previously, we produced a recombinant hyaluronidase antigenic fragment of Streptococcus pyogenes.

Objectives: This study aimed to improve the protein production and purity of hyaluronidase recombinant protein from S. pyogenes. In addition, the enzymatic activity of this protein was investigated.

Materialsand Methods:The expression of hyaluronidase antigenic fragments was optimized using IPTG concentration, time of induction, temperature, culture, and absorbance of 0.6-0.8-1 at $600 \mathrm{~nm}$. Afterwards, the expressed proteins were purified and the enzymatic activity was assessed by turbid metric method.

Results: Data indicated that maximum protein is produced in $\mathrm{OD}=0.8,0.5 \mathrm{mM}$ Isopropyl $\beta$-D-1-thiogalactopyranoside (IPTG), $37^{\circ} \mathrm{C}, \mathrm{NB}$ $1.5 \mathrm{x}$, without glucose, incubated for overnight. The enzymatic activity of the recombinant protein was similar to the commercial form of hyaluronidase.

Conclusions:The results showed that an antigenic fragment of the recombinant hyaluronidase protein from S. pyogenes has a considerable enzymatic activity. It can be suggested to use it for medical purposes. In addition, applications of bioinformatics software would facilitate the production of a smaller protein with same antigenic properties and enzymatic activity.

Keywords:Hyaluronidase; Optimization of Expression; Enzymatic Activity
\end{abstract}

\section{Background}

Hyaluronidases are enzymes that hydrolyze hyaluronic acid (HA) to N-acetyl-D-glucosamine (GlcNAc) and Dglucuronic acid (GlcA) in the body. The function of this enzyme is increasing the permeability of tissue to fluids (1). Hyaluronidase is produced in both prokaryotic and eukaryotic cells $(2,3)$. The catalytic mechanism of hyaluronidase is unknown (4); the structure and biological activity of hyaluronidase have been intensively studied in different microorganisms such as Streptococcus pyogenes (5), S. pneumoniae (6), Enterococcus (7), Clostridium perfringens (8), S. agalactiae (9), Pseudomonas aeruginosa (10) and Staphylococcus aureus (11).

Hyaluronidase in snake and insect venom is thought to function as a "spreading factor" by degrading the host hyaluronic acid, thus allowing the spread of toxin $(12,13)$. Therapeutic use of this enzyme is as a facilitating factor in the release of toxins and vaccine injected under the skin. Hyaluronidase has different pharmaceutical and medical applications (14) such as in ophthalmology (15), cancer (16), metastasis (17), tumor suppression (18), and fertilization (19) and also this enzyme can be used as a drug in combination with anesthesia and pain medication.

The hyaluronidase enzyme activity has been assayed by several methods including turbid metric (20), viscometer, microtiter-based enzyme linked immunosorbent assay (ELISA)-like assays $(21,22)$, colorimetric and fluorimetric assay, each of which have a particular function. For instance, colorimetric assay is used for determination of the serum hyaluronidase activity, fluorimetric assay is utilized for definition of biological samples such as human and rabbit serum hyaluronidase, and turbid metric and viscos metric methods are unsatisfactory in clinical use (23-27).

This enzyme has significant implications in different areas such as medical, diagnostic, treatment, physiologic, pathologic and commercial usage; future research and studying is implicated. It is important to produce a small

Copyright (C) 2015, Ahvaz Jundishapur University of Medical Sciences. This is an open-access article distributed under the terms of the Creative Commons Attribution-NonCommercial 4.0 International License (http://creativecommons.org/licenses/by-nc/4.0/) which permits copy and redistribute the material just in noncommercial usages, provided the original work is properly cited. 
and stable enzyme with same properties and effects. Therefore, in this study, the hyaluronidase antigenic fragments protein was overexpressed and purified in an appropriate condition then, the enzyme activity was measured using the turbid metric method.

\section{Objectives}

The main goal of our study was to optimize the production of hyaluronidase and improve the enzymatic properties of this enzyme in a smaller size.

\section{Materials and Methods}

\subsection{Designing, Gene Amplification, Cloning and Expression of Antigenic Fragment}

To find out the antigenic region, the sequence of hylA [S. pyogenes, accession number: EU078690.1] was submitted to Emboss antigenic server and the region with high antigenic properties was selected. Primers (CinnaGen, Iran) were designed according to the resulted sequences. Genomic DNA was extracted according to the standard $\mathrm{CTAB} / \mathrm{NaCl}$ method (28) and PCR (Roche, Germany) was used to synthesize the antigenic fragments of hyaluronidase. Afterwards, the fragment was cloned in pGEX-4T-1 expression vector (Pharmacia, Sweden) and expressed in Escherichia Coli BL21 (Pharmacia, Sweden) (29).

\subsection{Optimization of protein Expression and Puri- fication}

The expression of hyaluronidase antigenic fragment was optimized using different Isopropyl $\beta$-D-1thiogalactopyranoside (IPTG) (Merck, Germany) concentrations (0.5 and $1 \mathrm{mM}$ ), times of induction (two hours, four hours and overnight), temperature $\left(30^{\circ} \mathrm{C}\right.$ and $\left.37^{\circ} \mathrm{C}\right)$, culture media [NB (Merck, Germany) 1.5x without glucose containing $100 \mathrm{mg}$ ampicillin (Merck, Germany), NB (Merck, Germany) 1.5x with glucose (Merck, Germany) containing $100 \mathrm{mg}$ ampicillin (Merck, Germany), LuriaBertani (LB) broth (Merck, Germany) in one liter including 10 g yeast extract (Merck, Germany), 20 g Bacto tryptone broth (Merck, Germany), 0.2\% (mass/v) glucose (Merck, Germany), $10 \mathrm{~g} \mathrm{NaCl}$ (Roche, Germany), $1 \mathrm{~g} \mathrm{KCl}$ (Roche, Germany), $0.5 \mathrm{~g} \mathrm{MgCl}_{2}$ (Merck, Germany), $0.5 \mathrm{~g} \mathrm{CaCl}_{2}$ (Merck, Germany), $100 \mathrm{mg}$ ampicillin (Merck, Germany) and LB broth (Merck, Germany), and all the mentioned with the same concentration without glucose (Merck, Germany)] with 0.6-0.8-1 absorbance at $600 \mathrm{~nm}$. The expressed proteins were purified using GST-sepharose column according to the manufacture instruction (Sigma, Germany)(29).

\subsection{Determination of Protein Concentration}

The quality and quantity of the purified recombinant hyaluronidase proteins were assessed by sodium dodecyl sulfate polyacrylamide gel electrophoresis [SDS-PAGE (12\%)] with the absorbance of 280 and $260 \mathrm{~nm}$, respective- ly (28). Proteins concentration was measured using the following formula: Measurement of protein concentration $(\mathrm{mg} / \mathrm{mL})=(1.55 \times$ OD 280$)-(0.76 \times$ OD 260$)$

\subsection{Hyaluronidase Activity Assay}

The enzymatic assay of hyaluronidase antigenic fragments was determined as U/mg using a quantitative turbid metric method based on Dorfman, A(30). In principle, hyaluronic acid as substrate is hydrolyzed and depolymerized by hyaluronidase. Conditions of this method are $\mathrm{T}=37^{\circ} \mathrm{C}, \mathrm{pH}=5.9, \mathrm{~A} 600 \mathrm{~nm}$, light path $=1 \mathrm{~cm}$. Briefly, the recombinant protein was dissolved in $1 \mathrm{~mL}$ enzyme buffer (0.02 M phosphate buffer, 0.45\% $\mathrm{NaCl}$ (Roche, Germany), $0.01 \%$ bovine serum albumin (BSA) (Sigma, Germany), $\mathrm{pH}$ =6.8-7.0, mixed with $0.5 \mathrm{mg} \mathrm{HA}$ (Sigma, Germany), which was dissolved in $1 \mathrm{~mL}$ substrate butter (0.3 $\mathrm{M} \mathrm{KH}_{2} \mathrm{PO}_{4} /$ $\mathrm{Na}_{2} \mathrm{HPO}_{4}$ (Merck, Germany), $\mathrm{pH}=5.30-5.35$ ). Enzyme digestion was allowed to proceed for 45 minutes at $37^{\circ} \mathrm{C}$. Turbidity was generated at the end of the incubation by adding $10 \mathrm{~mL}$ acid albumin (Merck, Germany) solution. Optical density at $600 \mathrm{~nm}$ was determined exactly five minutes after the addition of acid albumin.

National formulary standard hyaluronidase solution (NF Std) (Sigma, Germany) was used as a standard sample in different concentrations and to establish a standard curve; units of activity were calculated by comparing them to this curve. A standard curve was made using commercial hyaluronidase (Sigma, Germany) with different known concentrations diluted from a stock of 400$1000 \mathrm{mg} / \mathrm{mL}$ as follows: $0.1,0.2,0.3,0.4,0.5$, and 0.6 .

\section{Results}

\subsection{Designing, Gene Amplification, Cloning and Expression of Antigenic Fragment}

According to the result of the server, the amino acid sequence of 838 till 2320 was selected as a region with high antigenic properties (Figure 1). The target gene was successfully extracted and amplified, then was cloned and expressed in pGEX-4T-1 expression vector (29).

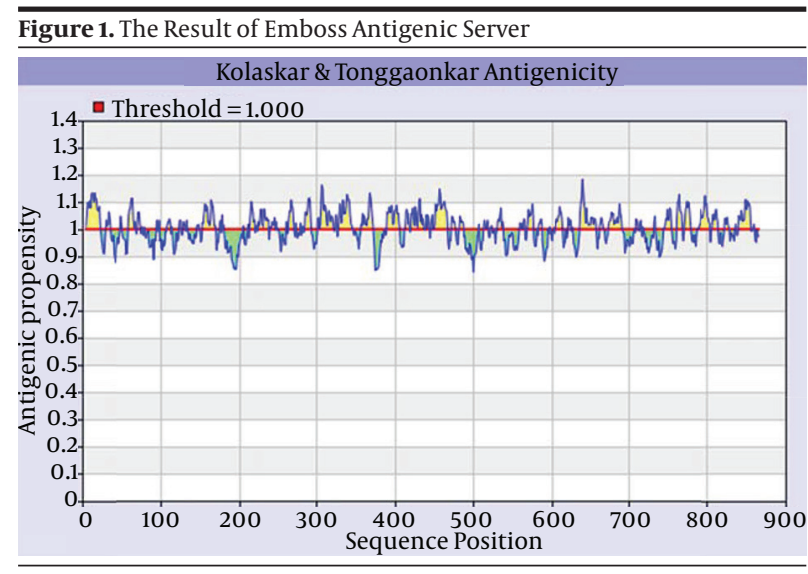

Average: 1.007; minimum: 0.846; maximum: 1.185; threshold: 1.000. 
Table 1. Yield of Hyaluronidase Obtained from pGEX4T1-hylA in Different Conditions of Protein Expression ${ }^{\text {a }}$

\begin{tabular}{|c|c|}
\hline Sample & Protein, $\mathrm{mg} / \mathrm{mL}$ \\
\hline HylA elute sample, 1 mM IPTG and OD $=1$ & 0.65 \\
\hline HylA elute sample, 0.5 mM IPTG and OD $=1$ & 0.7 \\
\hline HylA elute sample, 1 mM IPTG and $O D=0.8$ & 0.85 \\
\hline HylA elute sample, $0.5 \mathrm{mM}$ IPTG and OD = 0.8 & 0.93 \\
\hline HylA elute sample, $1 \mathrm{mM}$ IPTG and $\mathrm{OD}=0.6$ & 0.47 \\
\hline HylA elute sample, 0.5 mM IPTG and OD = 0.6 & 0.54 \\
\hline HylA elute sample, in NB 1.5x without glucose and induction at $37^{\circ} \mathrm{C}$ & 1.2 \\
\hline HylA elute sample, in NB $1.5 x$ without glucose and induction at $30^{\circ} \mathrm{C}$ & 0.82 \\
\hline HylA elute sample, in NB 1.5x with glucose and induction at $37^{\circ} \mathrm{C}$ & 0.9 \\
\hline HylA elute sample, in NB $1.5 x$ with glucose and induction at $370^{\circ} \mathrm{C}$ & 0.76 \\
\hline HylA elute sample, in LB broth with glucose and induction at $37^{\circ} \mathrm{C}$ & 0.6 \\
\hline HylA elute sample, in LB broth with glucose and induction at $30^{\circ} \mathrm{C}$ & 0.5 \\
\hline HylA elute sample, in LB broth without glucose and induction at $37^{\circ} \mathrm{C}$ & 0.75 \\
\hline HylA elute sample, in LB broth without glucose and induction at $30^{\circ} \mathrm{C}$ & 0.5 \\
\hline HylA elute sample, in $2 \mathrm{~h}$ of induction and $\mathrm{OD}=0.8$ & 0.4 \\
\hline HylA elute sample, in $4 \mathrm{~h}$ of induction and $\mathrm{OD}=0.8$ & 0.7 \\
\hline HylA elute sample, in overnight induction and $O D=0.8$ & 1.8 \\
\hline
\end{tabular}

a Abbreviations: NB, X; OD, optical density; LB, Luria-Bertani; IPTG, Isopropyl $\beta$-D-1-thiogalactopyranoside.

\subsection{Optimization of Gene Expression}

We changed the expression conditions of this protein to obtain the maximum expression. Afterwards, each recombinant protein was purified with affinity chromatography; then, the amount of purified recombinant protein was determined (Table 1 ).

\subsubsection{Effect of Glucose on HylA Production}

To evaluate the effect of glucose on tac-promoter activity, we added glucose to the culture media. According to Figure 2 and Table 1, expression of the antigenic fragments of hyaluronidase A in NB 1.5x without glucose had the highest protein expression levels compared with NB $1.5 x$ with glucose, LB broth with glucose, and LB broth without glucose. The results showed that although in lower glucose concentrations the tac-promoter activity will be increased, it will be followed by a reduced rate of protein production.

\subsubsection{Comparison of Temperature Induction}

Another important factor affecting protein expression was temperature shift. Hence, protein expression levels were determined at $30^{\circ} \mathrm{C}$ and $37^{\circ} \mathrm{C}$. The results suggest that the expression level of this recombinant protein in $37^{\circ} \mathrm{C}$ was higher than that in $30^{\circ} \mathrm{C}$, while the solubility of the expressed protein was equal in both temperatures, as

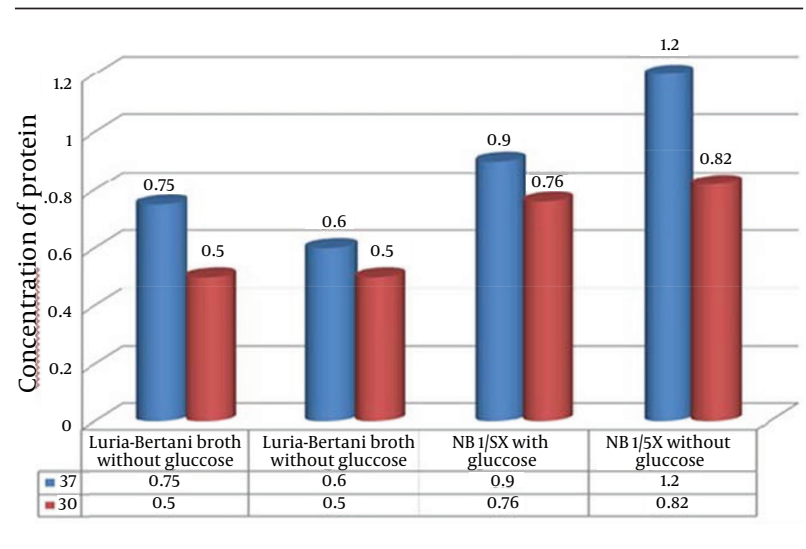

Figure 2. Amounts of Purified Recombinant Protein in Different Culture Media and Temperature Shifts $\left(30^{\circ} \mathrm{C}\right.$ and $\left.37^{\circ} \mathrm{C}\right)$

shown in Figure 2. This condition was used in all the induction experiments.

\subsubsection{Comparison of the Incubation Time on HylA Pro- duction}

To analyze the role of incubation time on protein expression, the samples were incubated at three different times. The data showed that the pick of expression level of antigenic fragments increased in 24 hours after induction in NB 1.5x without glucose (Figure 3). 


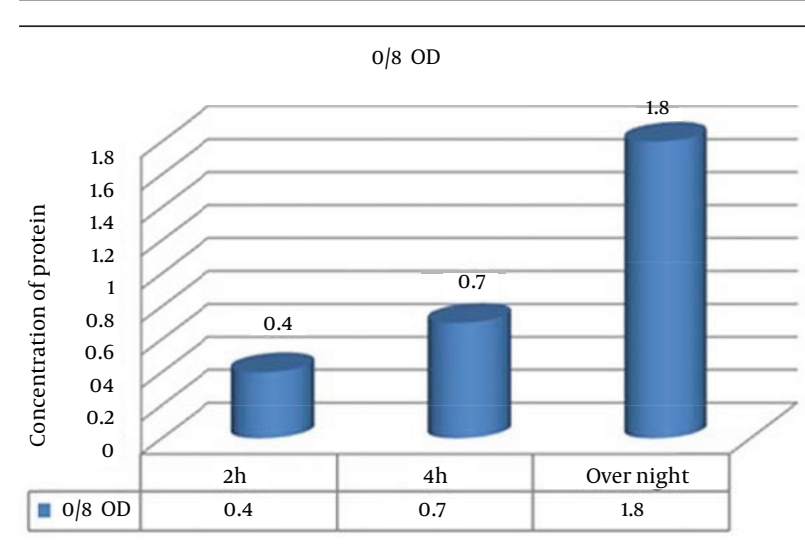

Figure 3. Amounts of Purified Recombinant Protein According to Time of Induction (Two hours, Four hours and overnight)

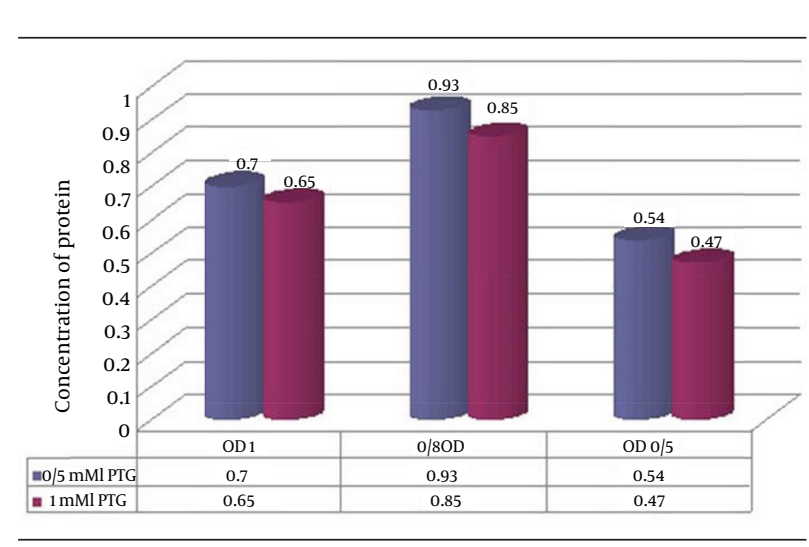

Figure 4. Amounts of Purified Recombinant Protein for Different Isopropyl $\beta$-D-1-Thiogalactopyranoside Concentrations (0.5 and $1 \mathrm{mM}$ ) and Different Optical Densities (0.6, 0.8 and 1)

\subsubsection{Effect of Isopropyl $\beta$-D-1-Thiogalactopyranoside Concentration on HylA Production}

Another parameter that affected the protein expression was IPTG concentration. Protein expression level in 0.5 mM IPTG was more effective than in $1 \mathrm{mM}$. Trends were determined using column regression analysis (Figure 4).

\subsubsection{Effect of Different Optical Densities on HylA Pro- duction}

As showed in Figure 3, the middle column has $\mathrm{OD}=0.8$ which is the optimum for enzyme expression. ODs of 0.6 and 1 are in two other columns in which the expression of the enzyme is reduced. A higher level of HylA concentration was found in $\mathrm{OD}=0.8$.

\subsection{Biological Assay}

To determine the enzymatic activity, recombinant protein turbid metric assay was performed. According to the standard curve from serial dilutions of a commercial hyaluronidase stock, the results revealed that the concentration of produced recombinant protein was about 0.77 $\mathrm{U} / \mathrm{mL}$ (Figure 5).

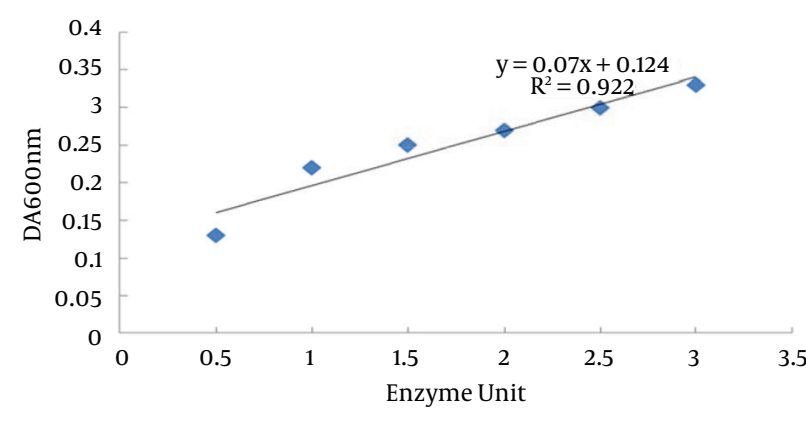

Figure 5. Quantitative Turbid Metric Assay for Determination of the Recombinant Hyaluronidase Activity. A standard curve was made using commercial hyaluronidase with different concentrations diluted from a stock of $1 \mathrm{mg} / \mathrm{mL}$. These concentrations were $3,2.5,1.5,1$, and $0.5 \mathrm{U} / \mathrm{mL}$.

\section{Discussion}

E. coli is well studied and its expression system is necessary for characterization of proteins. The maximum protein expression of bacteria is important for industrial purposes. There are many adjusting factors in the recombinant protein expression system of E. coli (31). Mello and colleagues applied esurience-related promoter to adjust the heterologous gene expression, once the culture got to the stationary phase (32). Galloway et al. and Chae et al. showed their efforts in inducing protein overexpression at late log or stationary phase $(33,34)$. Mahmoudi et al. performed initial experiments on chemical and temperature-inducible expression systems to identify appropriate expression conditions to improve the production of recombinant streptokinase. They showed that the amount of recombinant streptokinase increased in the medium containing $2.4 \%$ glucose, $480 \mu \mathrm{g} / \mathrm{mL}$ tryptone, $580 \mu \mathrm{g} / \mathrm{mL} \mathrm{MgSO}_{4}$ in $37^{\circ} \mathrm{C}$ (35). Jiang et al. explained the stationary phases of three E. coli systems, BL21 DE3 (pET), DH5a (pGEX) induced with lactose, and TG1 (pBV220) induced with heat shock, which could overexpress diversified genes, including three whose products are deleterious to the host cells. Their results offered a better strategy for recombinant protein preparation in E. coli (36).

In this study, we showed that an important advance in HylA yield can be obtained by changing the expression conditions. The culture medium ingredients have significant effects on the protein expression ability of this enzyme. Our investigations showed that the gene encoding for antigenic fragments of hyaluronidase has better protein expression ability in NB 1.5x without glucose. The presence of glucose leads to agglomeration of acetate, which is a cell growth inhibitor (38). In addition, lac operon in pGEX expression system is sensitive to glucose effects. When glucose is present, the genes for lactose metabolism are transcribed to a small magnitude. Furthermore, maximal transcription of the lac operon happens 
only when glucose is absent and lactose is present. The action of cyclic adenosine monophosphate (cAMP) and a catabolite activator protein produce this consequence. When glucose is absent, cAMP binds to the catabolite activator protein and transcription of the lac promoter will be increased by increment binding of T7 RNA polymerase to the lac promoter (37).

In the present study, we used E. coli BL21 DE3 which has cytoplasmic protease genes such as lon, ompT, degP and htpR. These genes contribute to overexpression of the recombinant protein (38). Our result showed that temperature had direct effects on enzyme expression. Higher temperature causes higher level of enzyme expression which might be due to increase in correct folding of target proteins. OD is another effective factor on the expression of enzyme. The tac operon induction after the stationary has been a better strategy for plasmid stability and preparation of recombinant protein synthesis system (33).

Finally, we found that IPTG concentration is the most effective variable, acting as trigger for gene transcription in lac operon. IPTG stimulates the beta-galactosidase activity. Addition of IPTG to the medium causes induction of T7 RNA polymerase activity for DNA transcription for production of a recombinant plasmid. More than $0.5 \mathrm{mM}$ IPTG causes cell lysis of E. coli BL21 DE3 which effects gene expression and formation of inclusion body (33). The commercial availability of hyaluronidase A has improved the diagnosis of S. pyogenes infection. Assessment of hyaluronidase activity is necessary due to its applications in diagnosis of diseases. Since the recombinant protein of the antigenic fragments of this enzyme has similar antigenic property and enzymatic activity, it can be used for diagnosis of S.pyogenes infection. Therefore, the necessity of new purified and effective recombinant hyaluronidase antigenic fragments is not only of academic importance but also of practical interest $(29,39)$.

The recombinant hyaluronidase can be assayed by spectrophotometric methods which detect the formation of an unsaturated bond during the catalysis of this enzyme. Stern et al. have engendered a novel ELISA-like rapid and easy assay for hyaluronidase. This method is based on a high affinity biotinylated HA-binding peptide derived from tryptic digests of proteoglycan core protein of bovine nasal cartilage and the avidin-biotin reaction. In this method, a standard curve was plotted for hyaluronidase activity, to which all unknown enzyme samples were compared (21). The colorimetric Morgan-Elson assay method is an insensitive method based on a reducing N-acetyl-D-glucosamine terminus (24). Takahashi et al. expressed a fluorimetric Morgan-Elson assay method for hyaluronidase activity. This method has high sensitivity, is rapid, and has a detection limit of 5x 10-3 NFU/ $\mathrm{mL}$ of bovine testicular hyaluronidase after one hour incubation. This method is more useful for measurement of low hyaluronidase activity in biological samples such as human and rabbit serum hyaluronidase (22). Chen et al. has described a new micro-method for assessment of hyaluronidase activity. This method is based on modified disc gel electrophoresis and chondroitin sulfate is used as a substrate. This is a sensitive method for assessment of hyaluronidase activity in serum and urine (23). Pearce et al. has explained that turbidimetric method is based on the turbidity-reducing activity of hyaluronidase in 10 minutes of incubation. This method is easy and cost effective and the hyaluronidase activity of most bacteria can be demonstrated (40).

The result of turbid metric assay demonstrated that the concentration of producing recombinant protein was about $0.77 \mathrm{U} / \mathrm{mL}$. The result of the present study indicated that this fragment had enzymatic activity; revealing the active site and antigenic dominance in the amino acid sequence of 838 to 2320 of this protein. On the other hand, the enzymatic activity of this recombinant protein was similar to commercial hyaluronidase. Hence, this fragment is a good candidate for producing large amounts of commercial recombinant hyaluronidase. In addition to antigenic properties, hyaluronidase antigenic fragments have an enzymatic activity similar to the whole gene. This matter can be used in molecular biology, particularly in further investigations, to obtain a small protein with both enzymatic and antigenic properties. Data indicated that the antigenic fragment of the recombinant hyaluronidase protein from S. pyogenes had considerable enzymatic activity and can be used for medical purposes.

\section{Acknowledgements}

The authors would like to thank the Deputy of Research and Technology of Arak University of Medical Sciences, Iran, for financial and equipment support.

\section{Authors' Contributions}

Shabnam Sadoogh Abbasian, Neda Akbari and Hamid Abtahi designed the research plan, organized the study, participated in all the experiments and coordinated the data analysis. Ehsanollah Ghaznavi-Rad, Mohammad Reza Zolfaghari and Iraj pakzad contributed to the writing of the manuscript.

\section{Funding/Support}

This study was supported by the Molecular and Medicine Research Center of Arak University of Medical Sciences, Iran.

\section{References}

1. Bisno AL, Brito MO, Collins CM. Molecular basis of group A streptococcal virulence. Lancet Infect Dis. 2003;3(4):191-200.

2. Douglas B, Muchmore MD, Daniel E. Review of the Mechanism of Action and Clinical Efficacy of Recombinant Human Hyaluronidase Coadministration with Current Prandial Insulin Formulations. J Diabetes Sci Tech. 2010:419-28.

3. Girish KS, Kemparaju K. The magic glue hyaluronan and its eraser hyaluronidase: a biological overview. Life Sci.2007;80(21):1921-43.

4. Stern R, Jedrzejas MJ. Hyaluronidases: their genomics, structures, and mechanisms of action. Chem Rev. 2006;106(3):818-39. 
5. Hynes WL, Dixon AR, Walton SL, Aridgides LJ. The extracellular hyaluronidase gene (hylA) of Streptococcus pyogenes. FEMS Microbiol Lett. 2000;184(1):109-12.

6. Park CY, Kim EH, Choi SY, Tran TD, Kim IH, Kim SN, et al. Virulence attenuation of Streptococcus pneumoniae clpP mutant by sensitivity to oxidative stress in macrophages via an NO-mediated pathway. J Microbiol. 2010;48(2):229-35.

7. Wu LX, Huang WX, Luo T, Sun XP. [Construction of hyaluronidase gene mutant of Enterococcus faecium and the function of hyaluronidase gene]. Zhonghua Yi Xue Za Zhi. 2007;87(40):2852-5.

8. Boraston AB, Ficko-Blean E, Healey M. Carbohydrate recognition by a large sialidase toxin from Clostridium perfringens. Biochemistry. 2007;46(40):11352-60.

9. Oettl M, Hoechstetter J, Asen I, Bernhardt G, Buschauer A. Comparative characterization of bovine testicular hyaluronidase and a hyaluronate lyase from Streptococcus agalactiae in pharmaceutical preparations. Eur J Pharm Sci. 2003;18(3-4):267-77.

10. Pierre G, Salah R, Gardarin C, Traikia M, Petit E, Delort AM, et al. Enzymatic degradation and bioactivity evaluation of C-6 oxidized chitosan. Int J Biol Macromol. 2013;60:383-92.

11. Abdelhak D. Alternative method for genetic transformation of Pasteurella multocida X73 using a hyaluronidase-producing Staphylococcus aureus strain. J Microbiol Methods. 2009;78(1):25-7.

12. Girish KS, Shashidharamurthy R, Nagaraju S, Gowda TV, Kemparaju K. Isolation and characterization of hyaluronidase a "spreading factor" from Indian cobra (Naja naja) venom. Biochimie. 2004;86(3):193-202.

13. Markovic-Housley Z, Miglierini G, Soldatova L, Rizkallah PJ, Muller U, Schirmer T. Crystal structure of hyaluronidase, a major allergen of bee venom. Structure. 2000;8(10):1025-35.

14. Aggarwal BB, Kumar A, Bharti AC. Anticancer potential of curcumin: preclinical and clinical studies. Anticancer Res. 2003;23(1A):363-98.

15. Hamada S, Devys JM, Xuan TH, Ganem S, Sahel JA, Heran F, et al. Role of hyaluronidase in diplopia after peribulbar anesthesia for cataract surgery. Ophthalmology. 2005;112(5):879-82.

16. Stern R. Hyaluronidases in cancer biology. Semin Cancer Biol. 2008;18(4):275-80.

17. Lokeshwar VB, Cerwinka WH, Isoyama T, Lokeshwar BL. HYAL1 hyaluronidase in prostate cancer: a tumor promoter and suppressor. Cancer Res. 2005;65(17):7782-9.

18. Kimura M, Kim E, Kang W, Yamashita M, Saigo M, Yamazaki T, et al. Functional roles of mouse sperm hyaluronidases, HYAL5 and SPAM1, in fertilization. Biol Reprod.2009;81(5):939-47.

19. Palte HD, Greenbaum S, Gayer S. Recombinant hyaluronidase. Clin Experiment Ophthalmol. 2014;42(3):298.

20. Girish KS, Jagadeesha DK, Rajeev KB, Kemparaju K. Snake venom hyaluronidase: an evidence for isoforms and extracellular matrix degradation. Mol Cell Biochem. 2002;240(1-2):105-10.

21. Stern M, Stern R. An ELISA-like assay for hyaluronidase and hyaluronidase inhibitors. Matrix. 1992;12(5):397-403.

22. Takahashi T, Ikegami-Kawai M, Okuda R, Suzuki K. A fluorimetric Morgan-Elson assay method for hyaluronidase activity. Anal Bio-

\section{chem. 2003;322(2):257-63.}

23. Chen SS, Hsu DS, Hoffman P. A new micro-assay for hyaluronidase activity. Clin Chim Acta.1979;95(2):277-84.

24. Rigouin C, Ladrat CD, Sinquin C, Colliec-Jouault S, Dion M. Assessment of biochemical methods to detect enzymatic depolymerization of polysaccharides. Carbohyd Polym. 2009;76: 279-284.

25. Jaques R. A Biological Method of Assay of hyaluronidase.London: Mill Hill; 1952.

26. Michael S, Robert S. An ELISA-Like Assay for Hyaluronidase and Hyaluronidase Inhibitors. Gustav Fischer Verlag. 1992:397-403.

27. Stair Nawy S, Csóka AB, Mio K, Stern R. Hyaluronidase Activity and Hyaluronidase Inhibitors: Assay Using a Microtiter-Based System. Meth Molecul Biol. 2001:383-9.

28. Sambrook J, Fritsch EF, Maniatis T. Molecular cloning: A Laboratory Manual. 3 edNew York: Cold Spring Harbor Laboratory; 2001.

29. Sadoogh Abbasian S, Abtahi H, Zolfaghari MR, Soufian S, Ghaznavi- Rad E. Cloning, expression, purification and antigenic evaluation of hyaluronidase antigenic fragments recombinant protein of streptococcus pyogenes. African J Biotech. 2012;11(9):2376-80.

30. Dorfman A. Methods in Enzymology. Mucopolysaccharidases 1955;1:166-73.

31. Kigawa T, Yabuki T, Matsuda N, Matsuda T, Nakajima R, Tanaka A, et al. Preparation of Escherichia coli cell extract for highly productive cell-free protein expression. J Struct Funct Genomics. 2004;5(1-2):63-8.

32. Mello BA, Tu Y. Effects of adaptation in maintaining high sensitivity over a wide range of backgrounds for Escherichia coli chemotaxis. Biophys J. 2007;92(7):2329-37.

33. Galloway CA, Sowden MP, Smith HC. Increasing the yield of soluble recombinant protein expressed in E. coli by induction during late log phase. Biotechniques. 2003;34(3):524-6-530.

34. Chae YK, Cho KS, Chun W, Lee K. Protein production by stationary phase induction (SPI). Protein Pept Lett. 2003;10(4):369-74.

35. Mahmoudi S, Abtahi H, Bahador A, Mosayebi G, Salmanian A. Teymuri M. Optimizing of nutrients for high level expression of recombinant streptokinase using pET32a expression system. JClinic Med. 2012;7(3).

36. Jiang X, Chen H, Yang W, Liu Y, Liu W, Wei J, et al. Functional expression and characterization of an acidic actinoporin from sea anemone Sagartia rosea. Biochem Biophys Res Commun. 2003;312(3):562-70.

37. Narang A, Pilyugin SS. Bistability of the lac operon during growth of Escherichia coli on lactose and lactose+glucose. Bull Math Biol. 2008;70(4):1032-64.

38. Shrestha P, Holland TM, Bundy BC. Streamlined extract preparation for Escherichia coli-based cell-free protein synthesis by sonication or bead vortex mixing. Biotechniques. 2012;53(3):163-74.

39. Hynes WL, Walton SL. Hyaluronidases of Gram-positive bacteria. FEMS Microbiol Lett. 2000;183(2):201-7.

40. Pearce RH. The turbidimetric estimation of hyaluronidase. Biochem J.1953;55(3):467-72. 